\title{
钝对二棕桐酰磷脂酰胆碱脂质体 相变性质的影响*
}

\author{
李新民张亚飞 倪嘉项 \\ (中国科学院长春应用化学研究所, 长春 130022) \\ 陈建文黄芬 \\ (中国科学院生物物理研究所, 北京 100080)
}

\section{关调 钝、二棕桐酰磷脂酰胆碱、相变}

随着稀土在农业、药物等方面的应用日益广泛, 特别是钝的二乙三胺五乙酸配合物（GdDTPA）已被临床应用于核磁共振成像的造影剂的等, 因此研究稀土在生物体内的作用机理, 已成为当前极感兴趣的课题, 虽然有关稀土的宏观毒理学已有不少报道 ${ }^{(2)}$, 但是研究稀土对生 物膜的影响报道很少 ${ }^{[3]}$, 钝对生物膜的作用尚未见报道, 稀土离子和钙离子在体内的竞争亦是 人们关心的问题, 本文采用差示扫描量热 (DSC)、激光拉曼技术研究了钝离子及 Gd-DTPA 等对人工膜二棕㭣酰磷脂酰胆碱 (DPPC) 脂质体相变性质的影响, 并观察了 $\mathrm{Gd}^{3+}$ 对人工膜 上结合钻的取代作用.

\section{一、材料和方法}

DPPC 系瑞士 Fluka 产品, $\mathrm{GdCl}_{3}$ 用 $99.99 \% \mathrm{Gd}_{2} \mathrm{O}_{3}$ 溶于盐酸除去过剩酸后, 用 EDTA 标定其浓度. Gd-DTPA 由 $\mathrm{Gd}_{2} \mathrm{O}_{3}$ 和 DTPA 反应制得, 其它试剂均为分析纯.

脂质体制备参考文献[4]. DSC 测定在 PE DSC-2C 型仪器上进行, 温度用铟标定, 升 温速率 $5 \mathrm{~K} / \mathrm{min}$, 微处理机检出相变温度 (以峰顶温度 $T_{m}$ 表示).

拉曼光谱在 SPEX-1403 双单色仪激光拉曼光谱仪上进行测定. $20^{\circ} \mathrm{C}$ 、激发线 $514.5 \mathrm{~nm}$, 氞离子激光器输出功率 $100 \mathrm{mw}$, 狭琏 $400 \mu \mathrm{m}$, 扫描速度 $0.5 \mathrm{~cm}^{-1} \cdot \mathrm{s}^{-1}$.

\section{二、结果和讨论}

1. $\mathrm{CaCl}_{2} 、 \mathrm{GdCl}_{3}$ 和 DPPC 作用的 DSC 研究 我们测得纯 DPPC 脂质体相变温 度 $\left(T_{m}\right)$ 为 $315.48 \mathrm{~K}$, 预相变温度为 $308.8 \mathrm{~K}$, 与文献 [5]报道相符.

$\mathrm{CaCl}_{2} 、 \mathrm{GdCl}_{3}$ 的加人使 DPPC 预相变峰消失, $\mathrm{DdCl}_{3}$ 使 CPPC 脂质体出现分相现象, 呈两个峰, 即高温部分有新峰出现 (图 1), 这可能是以 $\mathrm{Gd}^{3+}-\mathrm{DPPC}$ 为主要成分的相变峰, 而 加人 $\mathrm{CaCl}_{2}$ 只有在其与 DPPC 摩尔比较大时才使之分相. 随着氯化物浓度的增加, $T_{m}$ 逐 渐升高(图 1、表 1), 升高值由 $0.24 \mathrm{~K}$ 渐增至 $4.67 \mathrm{~K}$, 峰宽略有增大, 但峰形仍保持尖锐, 不失

本文 1990 年 12 月 10 日收到.

* 国家自然科学基金资助项目. 
怕变良好的协同性. 钝与钻的氧化物对 DPPC 脂质体相变温度亦即对膜流动性的作用程度 为: $\mathrm{GdCl}_{3}>\mathrm{CaCl}_{20}$. 因 $\mathrm{Ca}^{2+}$ 与 $\mathrm{Gd}^{3+}$ 的离子半径相近,但其电荷比 $\mathrm{Gd}^{3+}$ 小, 离子势小, 故对 离子型键合其强度小. $\mathrm{GdCl}_{3} 、 \mathrm{CaCl}_{2}$ 使 DPPC 脂质体相变温度开高, 说明使整个磷脂分子排 列的有序性提高, 膜流动性减小.

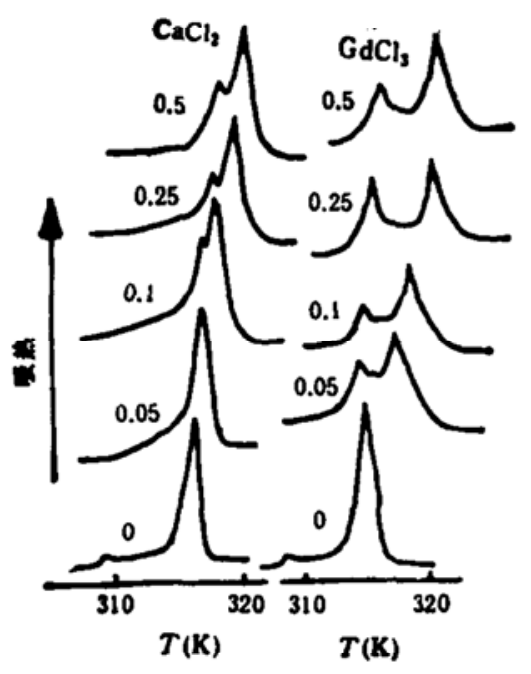

图 1 不同摩尔比 $\mathrm{CaCl}_{2} / \mathrm{DPPC}, \mathrm{GdCl}_{3} / \mathrm{DPPC}$ 对 DPPC DSC 热曲线的影响

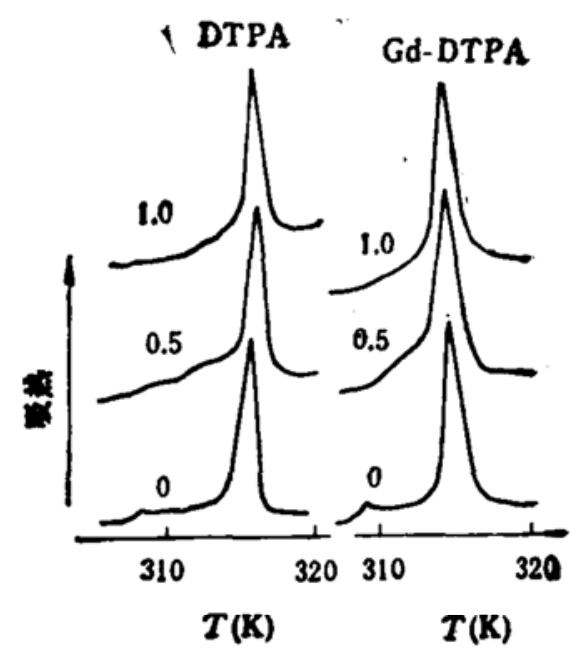

图 2 不同摩尔比 DTPA/DPPC、Gd-DTPA/ DPPC 对 DPPC DSC 热曲线的影响

表 1 钻和钟对 DPPC 脂质体相变温度 $T_{-}(\mathrm{K})$ 的影响

\begin{tabular}{|c|c|c|c|c|c|c|}
\hline DPPC & \multicolumn{6}{|c|}{ 尔比(物种/DPPC) } \\
\hline & 0 & 0.05 & 0.1 & 0.25 & 0.5 & 1.0 \\
\hline $\mathrm{CaCl}_{2}$ & 315.48 & 315.72 & 316.33 & 317.08 & 317.36 & \\
\hline $\mathrm{GdCl}_{3}$ & 315.48 & 317.72 & 319.12 & 320.05 & 320.15 & \\
\hline DTPA & 315.48 & & & & 315.52 & 315.47 \\
\hline Gd-DTPA & 315.48 & & & & 315.19 & 315.14 \\
\hline
\end{tabular}

2. DTPA、Gd-DTPA 和 DPPC 作用的 DSC 研究实验结果表明, 单独加入 DTPA 对 DPPC 相变温度无明显影响(表 1), 说明 DTPA 和 DPPC 脂质体之间并不发生 作用. 当钝离子和 DTPA 形成稳定配合物 Gd-DTPA 再与 DPPC 作用后, 从图 2、表 1 看 出, 对 DPPC 相变行为的影响比其氮化物小, 这说明 Gd-DTPA 中主要是少量解离后的钝离 子和 DPPC 脂质体作用而影响其相变性质.

3. Gd ${ }^{3+} 、 \mathrm{Gd}-\mathrm{DTPA}$ 和 DPPC 上 $\mathrm{Ca}^{2+}$ 竞争作用的 DSC 研究当 $\mathrm{Ca}^{2+}$ 和 DPPC 脂质体作用基本达到饱和时, 加人不同量的 $\mathrm{GdCl}_{3}$, 从图 3 可以看出, 其 DSC 曲线 特征与 $\mathrm{C}_{3} \mathrm{Cl}_{2}$ 和 DPPC 作用的 DSC 曲线明显不同, 而与 $\mathrm{GdCl}_{3}$ 和 DPPC 作用的 DSC 曲 线 (图 1)极为相似, 分相现象明显, 相变温度继续升高, 说明 $\mathrm{Gd}^{3+}$ 已取代 DPPC 上的 $\mathrm{Ca}^{2+}$. $\mathrm{Gd}^{3+}$ 能取代 $\mathrm{Ca}^{2+}$, 显然是由于 $\mathrm{Gd}^{3+}$ 配位能力比 $\mathrm{Ca}^{2+}$ 强之故. 从 $\mathrm{Gd}-\mathrm{DTPA}$ 和 DPPC 上 $\mathrm{Ca}^{2+}$ 竞争的 DSC 曲线(图 3)来看, Gd-DTPA 几乎对含有 $\mathrm{Ca}^{2+}$ 的 DPPC 脂质体浽有影 


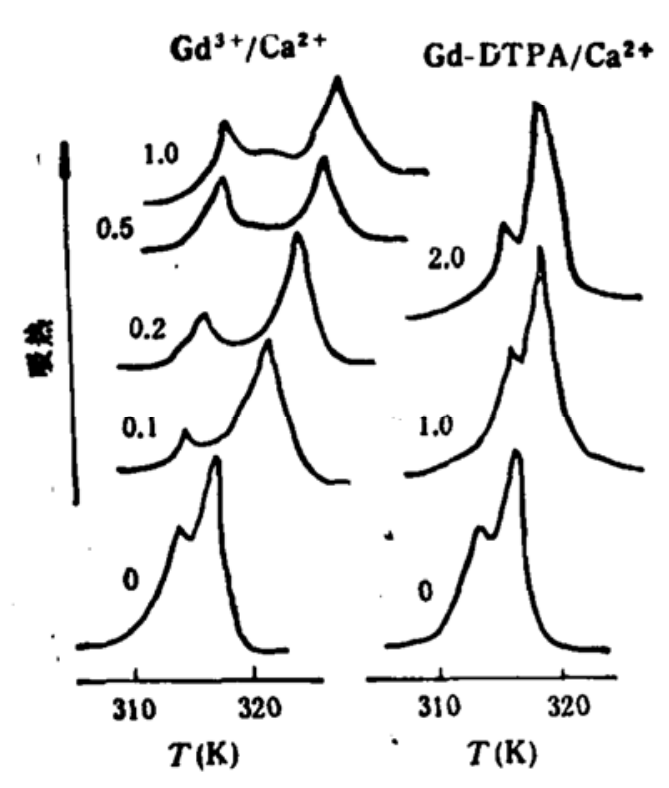

图 3 不同摩尔比 $\mathrm{Gd}^{3+} / \mathrm{Ca}^{2+} 、 \mathrm{Gd}-\mathrm{DTPA} / \mathrm{Ca}^{2+}$ 对 DPPC DSC 热曲线的影响

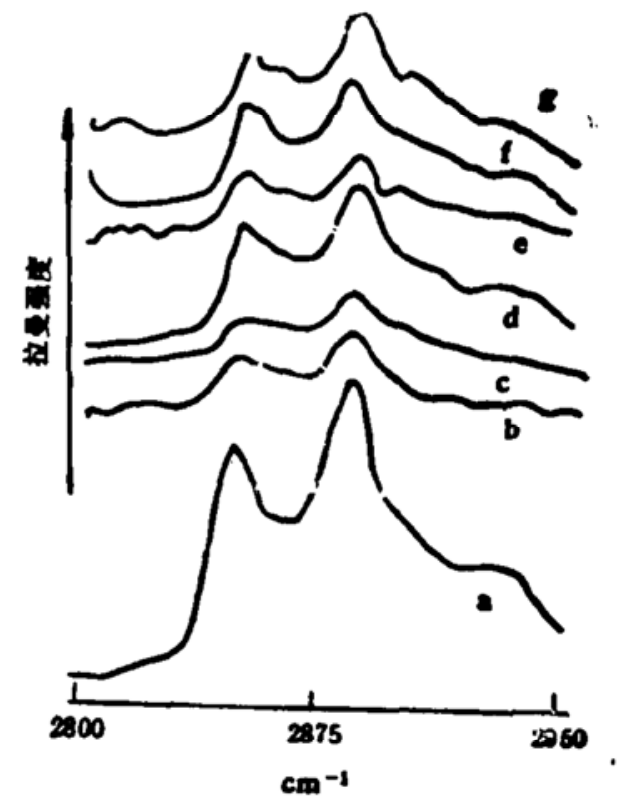

图 4 钝对 DPPC C-H区拉曼光谱的影呵

a. $\operatorname{DPPC}(\mathrm{S})$; b. $\operatorname{DPPC}(\mathrm{L}) ;$ c. $G \mathrm{GCl}_{3} / \mathrm{DPPC}=0.1$; d. $\mathrm{GdCl}_{3} / \mathrm{DPPC}=0.5 ;$ e. $\mathrm{DTPA} / \mathrm{DPPC}=0.5 ;$ f. $G d-D T P A / D P P C=0.5 ;$ g. Gd-DTPA $/ D P P C=1.0$

响,进一步说明当钝离子与配位能力强的配体配位后对生物膜的作用变小.

4. $\mathrm{GdCl}_{3}$ 和 DPPC 脂质体作用的拉复光请研究 为了研究 $\mathrm{GdCl}_{3}$ 对 DPPC 脂质 体构象的变化, 我们在室温 $\left(20^{\circ} \mathrm{C}\right)$ 测定了固态 DPPC、DPPC 脂质体及加人 $\mathrm{GdCl}_{3}$ 后的拉曼 光谱(图 4), 按文献 [6]常用的方法, 用 $I_{1095} / I_{1124}$ 和 $I_{2882} / I_{2848}, S_{T}$ 及 $S_{L}$ 分别描述脂酰链内结构 的有序性和脂酰链间侧向排列的有序性. 从表 2 可看出, $\mathrm{GdCl}_{3}$ 对 DPPC 脂酰链的影响较 小, $\mathrm{GdCl}_{3}$ 和 DPPC 摩尔比为 0.1 时, $I_{2882} / I_{2848}, S_{L}$ 减小, 说明 $\mathrm{GdCl}_{3}$ 的加入使脂酰链则向 耦合减弱, 侧向排列趋向无序, 讪运动性增加, 而其摩尔比为 0.5 时, $I_{2882} / I_{2848} 、 S_{L}$ 略有增加, 即脂酰链有序性反而增加, 这与 $\mathrm{CaCl}_{2}$ 对 DPPC 脂酰链影响结果一致叨. 由于 $\mathrm{Gd}^{3+}$ 和 DPPC 极性头部 $\mathrm{PO}_{2}^{-}$作用, 会引起位于 $1095 \mathrm{~cm}^{-1}$ 附近的 $\mathrm{PO}_{2}^{-}$振动强度变化, 故 $I_{1095} / I_{1124}$ 、 $S_{\boldsymbol{T}}$ 不能给出其有序性的明确信息.

5. DTPA、Gd-DTPA 和 DPPC 脂质体作用的拉曼光请研究从 DTPA、GdDTPA 和 DPPC 脂质体作用的拉曼光谱结果 (图 4、表 2) 可知, DTPA 对 DPPC 脂酰链侧 向作用没有明显影响, 和 DSC 法结果一致, Gd-DTPA 对 DPPC 脂酰链的影响较小.

表 2 钝对 DPPC 脂质体 C-C 和 C-H 伸缩振动强度的影响

\begin{tabular}{l|c|c|c|c}
\hline 物种 & $I_{1095} / I_{1124}$ & $S_{T}$ & $I_{2182} / I_{2848}$ & $S_{L}$ \\
\hline 固态 DPPC & 0.8543 & 1 & 1.2189 & 0.3460 \\
DPPC 脂质体 & 0.8976 & 0.9518 & 1.0599 & 0.2399 \\
$G d C l_{3} / D P P C=0.1$ & 0.8266 & 1.0335 & 1.0575 & 0.2383 \\
$G d C l_{3} / D P P C=0.5$ & 0.8590 & 0.9945 & 1.1083 & 0.2722 \\
DTPA/DPPC $=0.5$ & 0.8592 & 0.9943 & 1.0596 & 0.2397 \\
Gd-DTPA/DPPC $=0.5$ & 0.9267 & 0.9219 & 1.0247 & 0.2165 \\
Gd-DTPA/DPPC $=1.0$ & 0.9235 & 0.9251 & 1.0252 & 0.2168 \\
\hline
\end{tabular}


为了进一步了解稀土对生物膜作用的分子机制, 有关工作正在进行中.

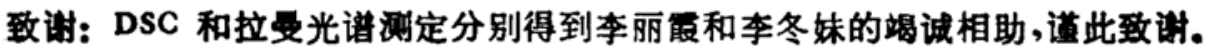

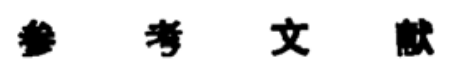

[1] Lauffer, R. B., Chem. Rev. 87(1987), 5: 901-927.

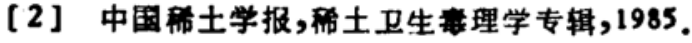

[3] Evans, C. H., Biochemistry of the Lanthanides, Plenum Press, New York, (1990).

[4] Huang Fen, Chen Jianwen and Fu Yigong et al., Kexwe Tongbao, 30(1985), 4: 539-543.

[5] 王苏民、英芬、傮亚珍等,科学通报,28(1983),1: 60-62.

[6] Gaber, B. P. and Peticolas, W. L, Biochim. Biophys. Acta, 465(1977), 260-274.

[7] Evgenija Loshchilora and Bele Karaly, Biochim. Biophys. Acte, 514(1978), 274-285. 